\title{
Locoregional Therapy for Cholangiocarcinoma
}

\author{
Nathan E. Frenk, MD ${ }^{1}$ Ronald S. Arellano, MD ${ }^{1}$ \\ ${ }^{1}$ Division of Interventional Radiology, Department of Radiology, \\ Massachusetts General Hospital, Harvard Medical School, \\ Boston, Massachusetts
}

\begin{abstract}
Address for correspondence Nathan Frenk, MD, Division of Interventional Radiology, Department of Radiology, Massachusetts General Hospital, Harvard Medical School, 55 Fruit Street, GRB 298, Boston, MA 02114 (e-mail: nfrenk@mgh.harvard.edu).
\end{abstract}

Dig Dis Interv 2017;1:47-52.

\author{
Abstract \\ Keywords \\ - locoregional therapy \\ - cholangiocarcinoma \\ - intrahepatic \\ cholangiocarcinoma \\ - thermal ablation \\ - transarterial \\ chemoembolization \\ - radioembolization
}

Cholangiocarcinoma represents the second most common primary hepatic malignancy and carries an overall poor prognosis. While surgical resection offers the only chances for cure, few patients are surgical candidates at the time of diagnosis. Chemotherapeutic treatment with gemcitabine and cisplatin is the standard chemotherapeutic regimen, but this offers only marginal overall survival benefits. With advances in minimally invasive techniques in the past decade, locoregional therapies with thermal ablation and transarterial techniques are increasingly being investigated as treatment options. This review explores emerging technologies and their potential role in treating cholangiocarcinoma.

Hepatic resection and transplantation are the only potentially curative options for cholangiocarcinoma. ${ }^{1,2}$ Unfortunately, only approximately $30 \%$ of patients are considered surgical candidates at the time of diagnosis and the overall 5 -year survival rates range from 33 to $65 \%{ }^{3}$ Given the dismal outcomes of systemic chemotherapy, other therapeutic options may have a potential role for patients with unresectable, recurrent, or metastatic disease. ${ }^{4,5}$ Thermal ablation or embolization may offer therapeutic options in selected patients with limited disease. Emerging early data suggest that by using principles of therapy used to treat other hepatic diseases, minimally invasive options may have a role in the treatment of selected cases of cholangiocarcinoma. ${ }^{6}$ This review explores emerging technologies and their potential role in treating cholangiocarcinoma.

\section{Thermal Ablation}

Thermal ablation has become a valuable treatment option for selected patients with primary or secondary hepatic tumors. Currently available thermal ablation options include radiofrequency ablation (RFA), microwave ablation (MWA), and cryoablation. These modalities have been used primarily for

received

December 6, 2016 accepted after revision

February 3, 2017

published online

March 20, 2017 the treatment of hepatocellular carcinoma (HCC), although some investigators have evaluated their role in the management of cholangiocarcinoma.

\section{Radiofrequency Ablation}

Radiofrequency ablation (RFA) is probably the most extensively used and investigated thermal ablation modality for the treatment of primary or metastatic liver tumors. RFA has shown clinical utility and acceptable tumor-free and overall survival benefits for selected patients with early stage HCC. ${ }^{6}$ Less extensively studied is the potential role and clinical benefit of RFA in management of cholangiocarcinoma. Nevertheless, emerging data suggest that the RFA may offer therapeutic benefits for intrahepatic cholangiocarcinoma (ICC). Early case reports describing intraoperative and percutaneous approaches were encouraging. In 2002, Slakey described the use of intraoperative RFA to treat a solitary intrahepatic recurrence that developed 12 months after resection of extrahepatic cholangiocarcinoma with right hepatic lobe involvement. Ten months after ablation, the patient remained free of detectable disease, thus raising the possibility that RFA might increase the number of patients considered surgically treatable. ${ }^{7}$ Similarly, Zgodizinksi and
Copyright $\odot 2017$ by Thieme Medical Publishers, Inc., 333 Seventh Avenue, New York, NY 10001, USA. Tel: +1(212) 584-4662.
DOI https://doi.org/ 10.1055/s-0037-1600117. ISSN 2472-8721.
Interventions; Guest Editor,

Ronald S. Arellano, MD 
Espat described a single case of RFA to treat a solitary ICC in a patient who remained tumor-free for 24 months after treatment. ${ }^{8}$ Chiou et al reported ultrasound (US)-guided percutaneous RFA of 10 histologically proven cholangiocarcinomas that ranged in size from 1.9 to $6.8 \mathrm{~cm}$ and found that complete tumor necrosis could be achieved in a single treatment session for all tumors less than $3 \mathrm{~cm}$ in size, and had only one complication after 12 treatment sessions for all tumors. ${ }^{9}$ Giorgio et al reported similar results in a cohort of 10 patients, including 9 patients with primary ICC and 1 recurrence after resection, treated with US-guided RFA. ${ }^{10}$ Local control has been reported as high as $89 \%$ for recurrent or primary ICC. ${ }^{11}$

\section{Cryoablation}

Limited clinical data exist on the use of cryoablation for treatment of cholangiocarcinoma. In general, cryoablation is used as one of the several palliative thermal ablation modalities for patients who are nonsurgical candidates. ${ }^{12}$ Most of the clinical data regarding cryoablation are embedded within studies that report the use of cryoablation for treatment of liver metastases. In a retrospective analysis of 57 patients with metastatic liver disease (41 colorectal and 16 non- colorectal) treated with cryoablation, Sheen et al used cryoablation to treat 57 patients with liver metastases and found that the median survival for patients with noncolorectal metastases was 37 months, compared with 22 months for patients with colorectal metastases. ${ }^{13}$

\section{Microwave Ablation}

Microwave ablation (MWA) is emerging as an alternative to RFA for locoregional ablative therapy for primary and secondary liver malignancies (- Fig. 1). ${ }^{12}$ The role of MWA for treatment of cholangiocarcinoma is yet to be defined, but early reports suggest clinical feasibility and safety. ${ }^{14,15}$, $\mathrm{Xu}$ et al reported overall survival rates for primary ICC of $87.5 \%, 75 \%, 62.5 \%$, and $62.5 \%$ at $6,12,36$, and 50 months, respectively. ${ }^{14}$ Yu et al reported cumulative overall 6-, 12-, and 24 -month survival rates of $78.7 \%, 60 \%$, and $60 \%$, respectively. ${ }^{15}$ Similar survival rates were reported by Yang et al, who retrospectively evaluated 26 patients with advanced ICC treated with MWA and transarterial chemoembolization (TACE) and observed 6-, 12-, and 24-month survival rates of $88.5 \%, 69.2 \%$, and $61.5 \%$, respectively. Additionally, the overall complete ablation rate was reported as $92 \%$ with no major treatment-related complications. ${ }^{16}$
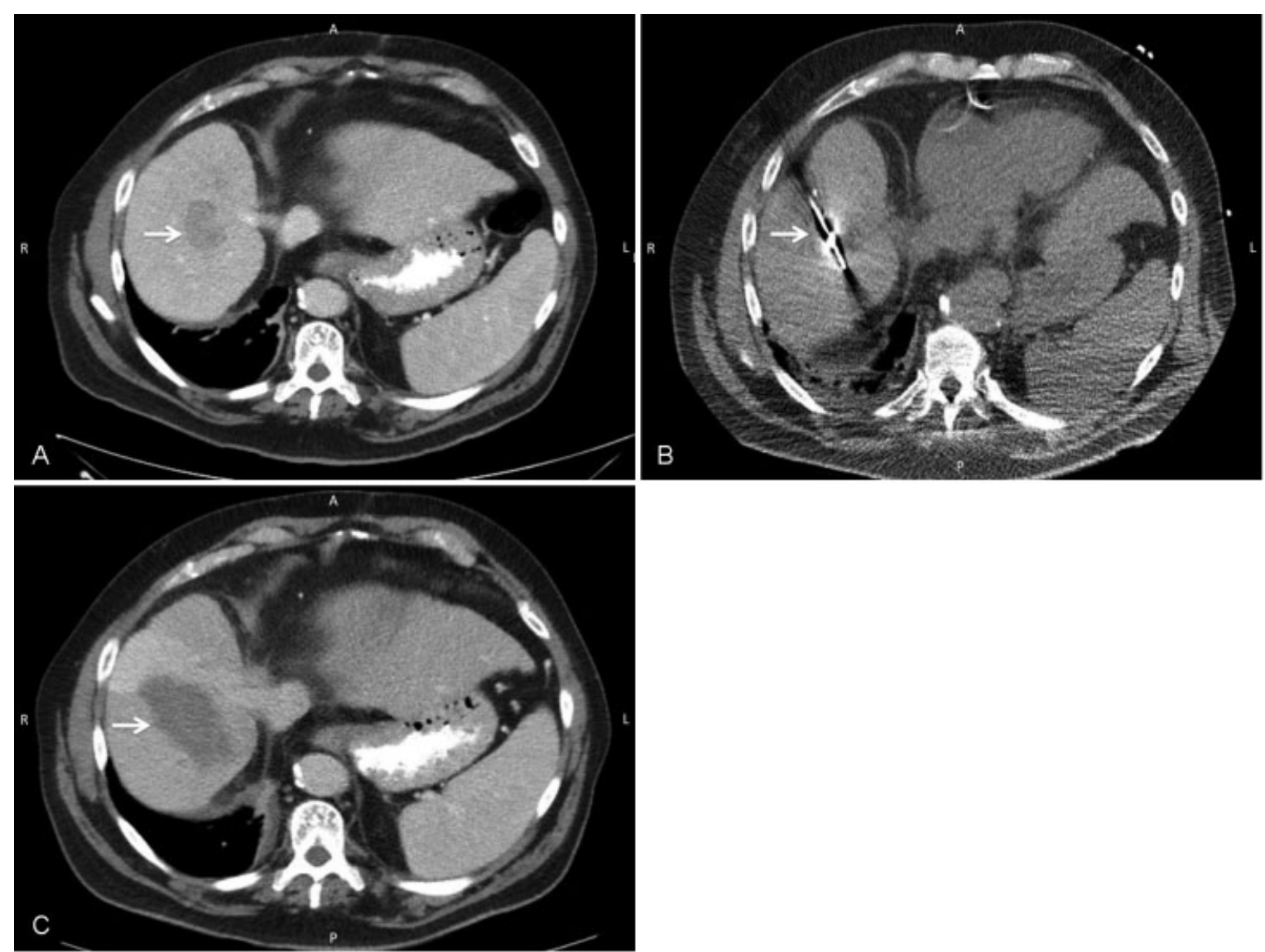

Fig. 1 (A) Axial contrast-enhanced CT scan of the abdomen in a 75-year-old with recurrent intrahepatic cholangiocarcinoma (white arrow). (B) Axial unenhanced CT scan of the abdomen in a 75-year-old with recurrent intrahepatic cholangiocarcinoma. White arrow indicates microwave antenna within the tumor at time of microwave ablation (white arrow). (C) Axial contrast material-enhanced CT scan of the abdomen in a 75-yearold with recurrent intrahepatic cholangiocarcinoma. White arrow indicates post-MWA zone of ablation of ICC 1 month after ablation. No residual tumor was detected. 


\section{Embolization Techniques}

Recurrence of cholangiocarcinoma after surgery is reportedly as high as $53 \%$, with most recurrences developing within the liver. ${ }^{17}$ The role of transarterial therapies for their treatment is somewhat controversial, with most literature reporting some effectiveness as a palliative technique. ${ }^{18-20}$ In a multi-institutional analysis of intra-arterial therapy for advanced ICC, Hyder et al evaluated the safety and efficacy of TACE versus bland embolization versus radioembolization. Assessment of tumor response based on RECIST (Response Evaluation Criteria in Solid Tumors) criteria showed partial response in $21.5 \%$ patients whereas $61.5 \%$ patients had stable disease and only $13 \%$ showed progressive disease. Additionally, overall survival was no different between the various modalities and ranged from 10.5 to 14.3 months. ${ }^{17}$ Park et al compared TACE versus supportive care as palliative treatment in 155 patients with unresectable ICC and showed that TACE was associated with significantly improved survival of 12.2 months compared with supportive care alone. ${ }^{21}$ A preliminary study comparing the efficacy of drug-eluting beads preloaded with irinotecan (DEBIRI) or doxorubicin (DEBDOX) as second-line treatment for unresectable metastatic cholangiocarcinoma suggested a slightly overall survival benefit with DEBIRI, though the progression-free survival was 12.7 weeks with DEBIRI versus 15.8 weeks for DEBDOX. ${ }^{22}$ Overall, emerging data suggest that the best role for TACE is as a palliative technique with modest survival benefits compared with systemic therapies with acceptable safety profiles. $^{23}$

\section{Radioembolization}

Selective internal radiation therapy (SIRT) using yttrium90-labeled microspheres is an emerging therapeutic option for management of cholangiocarcinoma ${ }^{24}$ (-Fig. 2). Radioembolization is typically indicated when surgery, chemotherapy, or local thermal ablation are not therapeutic options. Radioembolization is a multistep procedure: (1) A baseline positron emission tomography-computed tomography (PET-CT) is obtained to document fluorine-18-deoxyglucose (FDG) activity of the hepatic lesion(s) and assess for extrahepatic disease. It is also critical for the evaluation of posttreatment results. ${ }^{25-27}$ The CT of the PET-CT study can also be used for tumor volumetric analysis that is required for calculating the dose of yttrium-90 to be administered. (2) Pre-SIRT visceral angiography is necessary to map the hepatic arterial anatomy and assess tumor vascularity. If
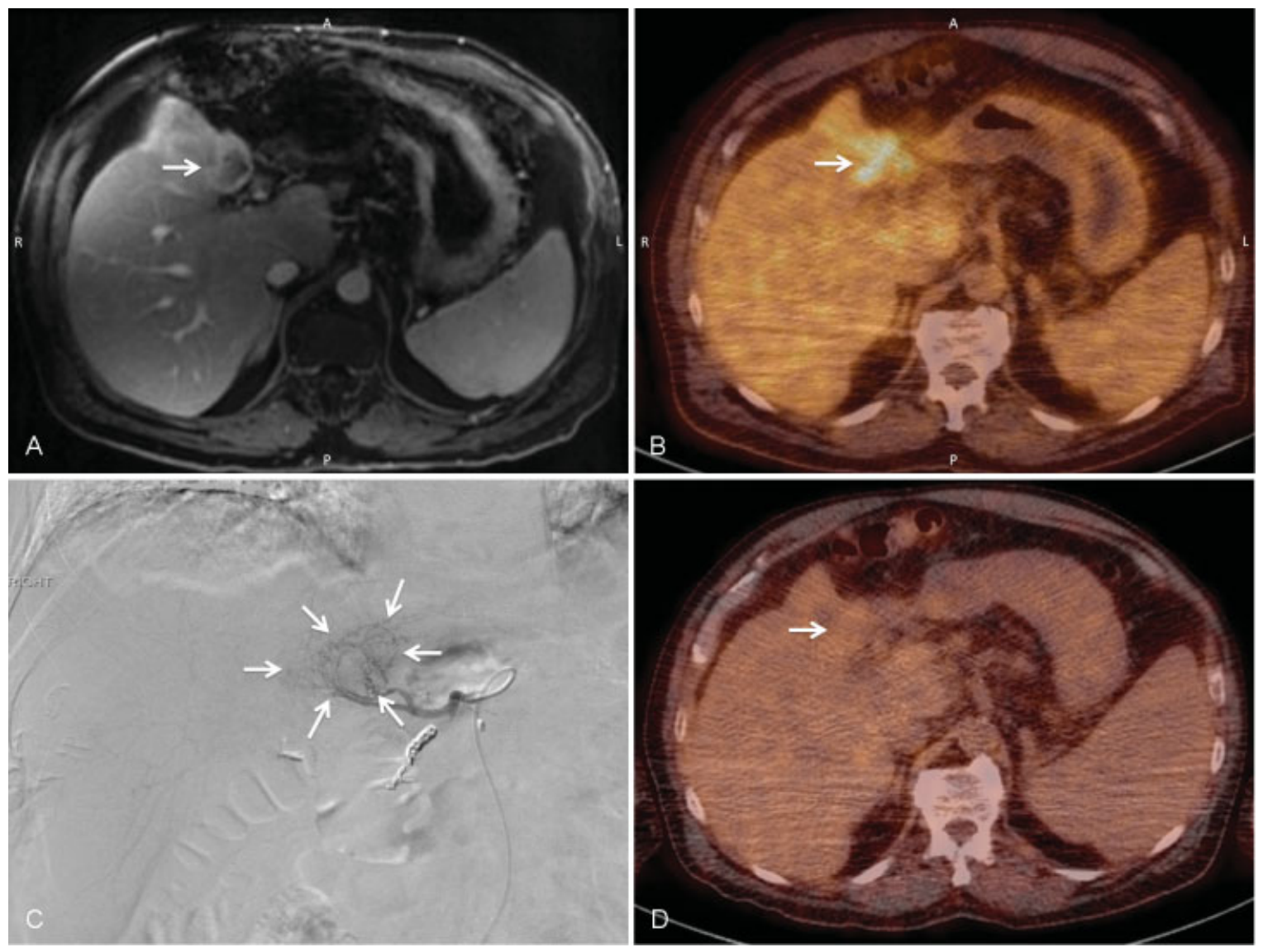

Fig. 2 (A) Axial gadolinium-enhanced MRI of the liver that demonstrates intrahepatic cholangiocarcinoma (white arrow). (B) Positron emission tomography-computed tomography (PET-CT) of intrahepatic cholangiocarcinoma that demonstrates focal area of increased FDG avidity that corresponds to tumor detected on MRI in - Fig. 2A. (C) Digital subtraction hepatic arteriography at time of selective internal radiation therapy that demonstrates tumor blush (white arrows). (D) PET-CT of a 75-year-old with intrahepatic cholangiocarcinoma obtained 2 months following radioembolization. White arrow indicates absent FDG avidity in segment 4 that was FDG-avid prior to treatment (-Fig. 2B). 
necessary, deliberate embolization of the branches of the hepatic artery such as the gastroduodenal, superior pancreaticoduodenal, or right gastric arteries is performed to minimize the risk of nontarget embolization. Intra-arterial injection of technetium-99m macroaggregated albumin (MAA) is then given to assess for extrahepatic shunts. In general, patients with hepatopulmonary shunt fractions less than $20 \%$ are suitable for selective internal radiotherapy (SIRT). (3) A second visceral angiogram is then performed for selective delivery of yttrium-90 microspheres. For patients with bilobar disease, the pre-SIRT and SIRT procedures are usually performed separately for each lobe.

Emerging clinical data suggest promising results with SIRT for treating ICC. ${ }^{28-32}$ Prognostic factors such as tumor size, FDG avidity, and tumor burden appear to impact the effectiveness of radioembolization. Patients with lower tumor load, FDG-negative tumors, and smaller tumors appear to survive longer with radioembolization. ${ }^{32,33}$ Mosconi et al evaluated the use of yttrium-90 radioembolization for unresectable or recurrent ICC and showed that SIRT was associated with significantly longer survival in treatment-naïve patients compared with patients who underwent previous surgery or other treatments. $^{32}$ The combination of radioembolization and systemic chemotherapy may also have a role in downstaging initially unresectable patients to resection candidates. Rayar et al recently published a series of 49 patients with unresectable ICC treated with yttrium-90 and chemotherapy. Ten of these patients were considered as potentially resectable of whom 8 eventually underwent R0 resections. ${ }^{34}$ A metaanalysis of 12 studies with relevant data regarding SIRT showed that overall survival of patients with ICC treated with radioembolization is higher than historical survival rates and similar survival to patients treated with standard chemotherapy and/or TACE. ${ }^{35}$

\section{References}

1 Schmassmann A. Nonsurgical therapies for hepatocellular and cholangiocellular carcinoma. Swiss Surg 1999;5(3):116-121

2 Scheuermann U, Kaths JM, Heise M, et al. Comparison of resection and transarterial chemoembolisation in the treatment of advanced intrahepatic cholangiocarcinoma-a single-center experience. Eur J Surg Oncol 2013;39(6):593-600

3 Kuhlmann JB, Blum HE. Locoregional therapy for cholangiocarcinoma. Curr Opin Gastroenterol 2013;29(3):324-328

4 Knüppel M, Kubicka S, Vogel A, et al. Combination of conservative and interventional therapy strategies for intra- and extrahepatic cholangiocellular carcinoma: a retrospective survival analysis. Gastroenterol Res Pract 2012;2012:190708

5 Park HM, Yun SP, Lee EC, et al. Outcomes for patients with recurrent intrahepatic cholangiocarcinoma after surgery. Ann Surg Oncol 2016;23(13):4392-4400

6 Livraghi T, Meloni F, Di Stasi M, et al. Sustained complete response and complications rates after radiofrequency ablation of very early hepatocellular carcinoma in cirrhosis: is resection still the treatment of choice? Hepatology 2008;47(1):82-89

7 Slakey DP. Radiofrequency ablation of recurrent cholangiocarcinoma. Am Surg 2002;68(4):395-397

8 Zgodzinski W, Espat NJ. Radiofrequency ablation for incidentally identified primary intrahepatic cholangiocarcinoma. World J Gastroenterol 2005;11(33):5239-5240
9 Chiou YY, Hwang JI, Chou YH, Wang HK, Chiang JH, Chang CY. Percutaneous ultrasound-guided radiofrequency ablation of intrahepatic cholangiocarcinoma. Kaohsiung J Med Sci 2005;21(7): 304-309

10 Giorgio A, Calisti G, DE Stefano G, et al. Radiofrequency ablation for intrahepatic cholangiocarcinoma: retrospective analysis of a single centre experience. Anticancer Res 2011;31(12):4575-4580

11 Butros SR, Shenoy-Bhangle A, Mueller PR, Arellano RS. Radiofrequency ablation of intrahepatic cholangiocarcinoma: feasability, local tumor control, and long-term outcome. Clin Imaging 2014;38(4):490-494

12 Amini A, Gamblin TC. Palliation: treating patients with inoperable biliary tract and primary liver tumors. Surg Oncol Clin N Am 2014;23(2):383-397

13 Sheen AJ, Poston GJ, Sherlock DJ. Cryotherapeutic ablation of liver tumours. Br J Surg 2002;89(11):1396-1401

14 Xu HX, Wang Y, Lu MD, Liu LN. Percutaneous ultrasound-guided thermal ablation for intrahepatic cholangiocarcinoma. Br J Radiol 2012;85(1016):1078-1084

15 Yu MA, Liang P, Yu XL, et al. Sonography-guided percutaneous microwave ablation of intrahepatic primary cholangiocarcinoma. Eur J Radiol 2011;80(2):548-552

16 Yang GW, Zhao Q, Qian S, et al. Percutaneous microwave ablation combined with simultaneous transarterial chemoembolization for the treatment of advanced intrahepatic cholangiocarcinoma. Onco Targets Ther 2015;8:1245-1250

17 Hyder O, Hatzaras I, Sotiropoulos GC, et al. Recurrence after operative management of intrahepatic cholangiocarcinoma. Surgery 2013;153(6):811-818

18 Herber S, Otto G, Schneider J, et al. Transarterial chemoembolization (TACE) for inoperable intrahepatic cholangiocarcinoma. Cardiovasc Intervent Radiol 2007;30(6):1156-1165

19 Shariff MI, Khan SA, Westaby D. The palliation of cholangiocarcinoma. Curr Opin Support Palliat Care 2013;7(2):168-174

20 Seidensticker R, Seidensticker M, Doegen K, et al. extensive use of interventional therapies improves survival in unresectable or recurrent intrahepatic cholangiocarcinoma. Gastroenterol Res Pract 2016;2016:8732521

21 Park SY, Kim JH, Yoon HJ, Lee IS, Yoon HK, Kim KP. Transarterial chemoembolization versus supportive therapy in the palliative treatment of unresectable intrahepatic cholangiocarcinoma. Clin Radiol 2011;66(4):322-328

22 Venturini M, Sallemi C, Agostini G, et al. Chemoembolization with drug eluting beads preloaded with irinotecan (DEBIRI) vs doxorubicin (DEBDOX) as a second line treatment for liver metastases from cholangiocarcinoma: a preliminary study. Br J Radiol 2016; 89(1067):20160247

23 Ray CE Jr, Edwards A, Smith MT, et al. Metaanalysis of survival, complications, and imaging response following chemotherapybased transarterial therapy in patients with unresectable intrahepatic cholangiocarcinoma. J Vasc Interv Radiol 2013;24(8): 1218-1226

24 Ahmadzadehfar H, Biersack HJ, Ezziddin S. Radioembolization of liver tumors with yttrium-90 microspheres. Semin Nucl Med 2010;40(2):105-121

25 Sharma B, Martin A, Zerizer I. Positron emission tomographycomputed tomography in liver imaging. Semin Ultrasound CT MR 2013;34(1):66-80

26 Camacho JC, Kokabi N, Xing M, Schuster DM, Kim HS. PET response criteria for solid tumors predict survival at three months after intra-arterial resin-based 90 yttrium radioembolization therapy for unresectable intrahepatic cholangiocarcinoma. Clin Nucl Med 2014;39(11):944-950

27 Filippi L, Pelle G, Cianni R, Scopinaro F, Bagni O. Change in total lesion glycolysis and clinical outcome after (90)Y radioembolization in intrahepatic cholangiocarcinoma. Nucl Med Biol 2015;42(1):59-64

28 Saxena A, Bester L, Chua TC, Chu FC, Morris DL. Yttrium-90 radiotherapy for unresectable intrahepatic cholangiocarcinoma: 
a preliminary assessment of this novel treatment option. Ann Surg Oncol 2010;17(2):484-491

29 Mouli S, Memon K, Baker T, et al. Yttrium-90 radioembolization for intrahepatic cholangiocarcinoma: safety, response, and survival analysis. J Vasc Interv Radiol 2013;24(8):1227-1234

30 Kloeckner R, Ruckes C, Kronfeld K, et al. Selective internal radiotherapy (SIRT) versus transarterial chemoembolization (TACE) for the treatment of intrahepatic cholangiocellular carcinoma (CCC): study protocol for a randomized controlled trial. Trials 2014;15:311

31 Kuei A, Saab S, Cho SK, Kee ST, Lee EW. Effects of yttrium-90 selective internal radiation therapy on non-conventional liver tumors. World J Gastroenterol 2015;21(27):8271-8283

32 Mosconi C, Gramenzi A, Ascanio S, et al. Yttrium-90 radioembolization for unresectable/recurrent intrahepatic cholangiocarci- noma: a survival, efficacy and safety study. Br J Cancer 2016; 115(3):297-302

33 Soydal C, Kucuk ON, Bilgic S, Ibis E. Radioembolization with (90)Y resin microspheres for intrahepatic cholangiocellular carcinoma: prognostic factors. Ann Nucl Med 2016;30(1): 29-34

34 Rayar M, Sulpice L, Edeline J, et al. Intra-arterial yttrium-90 radioembolization combined with systemic chemotherapy is a promising method for downstaging unresectable huge intrahepatic cholangiocarcinoma to surgical treatment. Ann Surg Oncol 2015;22(9):3102-3108

35 Al-Adra DP, Gill RS, Axford SJ, Shi X, Kneteman N, Liau SS. Treatment of unresectable intrahepatic cholangiocarcinoma with yttrium-90 radioembolization: a systematic review and pooled analysis. Eur J Surg Oncol 2015;41(1):120-127 